Research Article

\title{
The Drag Forces on a Taylor Bubble Rising Steadily in Vertical Pipes
}

Abdullah Abbas Kendoush *

Department of Nuclear Engineering Technology, Augusta Technical College, Augusta, GA, 30906, USA; E-Mail: akendoush@augustatech.edu

* Correspondence: Abdullah Abbas Kendoush; E-Mail: akendoush@augustatech.edu

Academic Editor: Faik Hamad

Special Issue: Multi-phase Flow with and without Heat Transfer

Journal of Energy and Power Technology

2021, volume 3 , issue 4

doi:10.21926/jept.2104048
Received: October 04, 2021

Accepted: November 28, 2021

Published: December 07, 2021

\begin{abstract}
By the adoption of a drag-buoyancy equality model, analytical solutions were obtained for the drag coefficients $\left(C_{D}\right)$ of Taylor bubbles rising steadily in pipes. The obtained solutions were functions of the geometry of the Taylor bubble and the gas volume fraction. The solutions were applicable at a wide range of Capillary numbers. The solution was validated by comparison with experimental data of other investigators. All derived drag formulas were subject to the condition that Bond number $>4$, for air-water systems.
\end{abstract}

\section{Keywords}

Slug flow; Taylor bubble; drag coefficient; two-phase flow

\section{Introduction}

Taylor bubbles characterize the slug flow pattern of two-phase gas-liquid flows in pipes. These are long gas bubbles with bullet-shaped nose, separated by slugs of liquid filling the entire cross-

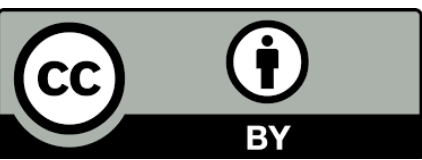

(C) 2021 by the author. This is an open access article distributed under the conditions of the Creative Commons by Attribution License, which permits unrestricted use, distribution, and reproduction in any medium or format, provided the original work is correctly cited. 
section of the pipe. This flow pattern occurs in several industrial applications, such as the steam generator of the pressurized water rector, boilers, petroleum industry, desalination processes, etc...

Steam-filled Taylor bubbles can sometimes collapse due to the injection of subcooled water and thus creating a water hammer with destructive consequences. Accident of this type occurred in San Onofre nuclear power plant in 1985 [1].

Two types of Taylor bubble shapes are encountered, in practice, one with a round (hemispherical) leading head and an almost flat trailing end seen in the low viscosity liquids, and the other has a spherical leading and trailing ends, seen in highly viscous liquids as shown in Figure 1. These phenomena were clarified and fully explained by the results of the experiments of Campos and Guedes De Carvalho [2]. The steady state rise terminal velocity of the Taylor bubble in stagnant liquid is given by the following equation of Dumitrescu [3]

$$
U=0.4949 \sqrt{g R}
$$

where $R$ is the tube radius and $g$ is the acceleration due to gravity. The liquid viscosity was neglected in the development of this equation, because it was based on inertia-dominated regime. Davies and Taylor's [4] equation was similar to Eq. (1) but the constant was 0.464.

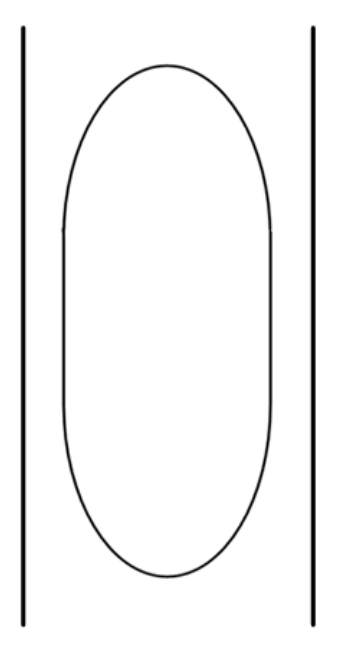

(a)

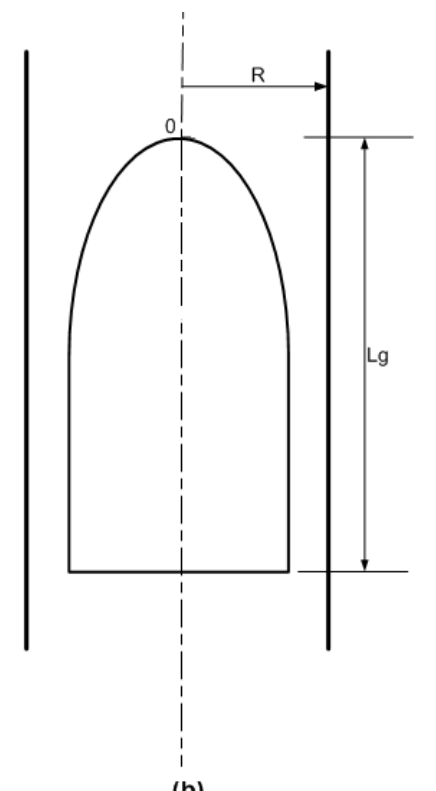

(b)

Figure 1 The two types of Taylor bubbles rising in (a) highly viscous liquid. and (b) Less viscous liquid.

Zukoski [5] and White and Beardmore [6] confirmed the applicability of Eq. (1). Clanet et al. [7] indicated that Eq. (1) is limited to Bond number $(B o>4)$ for air-water systems. Here $B o=$ $R / \sqrt{\sigma / \rho g}$. Where $\sigma$ is the surface tension, $\rho$ is the density of the liquid, and $g$ is the acceleration due to gravity.

Ishii and Mishima [8] obtained the following equation for the drag coefficient of the Taylor bubble in slug flow

$$
C_{D}=9.8(1-\alpha)^{3}
$$


Here $\alpha$ is the gas volume fraction. This equation was not validated by any investigator, as far as the author is aware. In this equation $C_{D}$ decreases with volume fraction, contrary to the author's results of the increase of $C_{D}$ with the increase in the interfacial surfaces of spherical bubbles [9]. It should be noted that $C_{D}$ of this equation does not depend on liquid properties.

Bretherton [10] derived the following equation for estimating the thickness $(m)$ of the thin liquid layer between the inside tube wall and the bubble surface (see Figure 2).

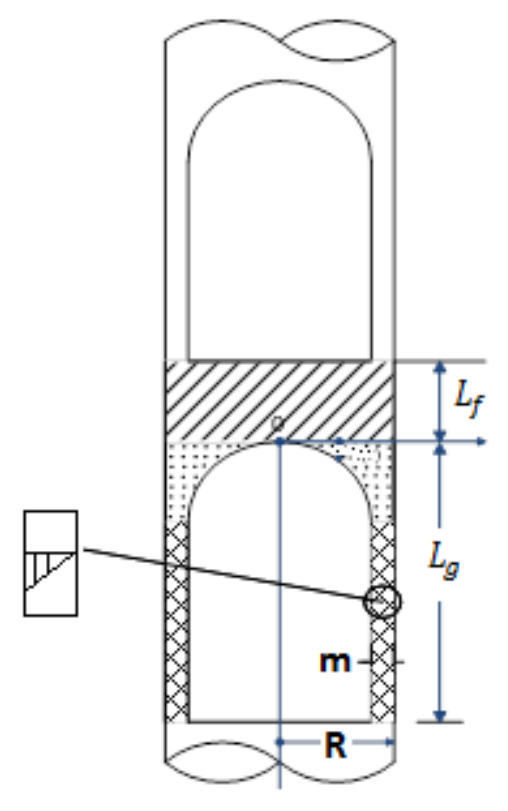

Figure 2 Partitions of the various flow regions around the flat-ended Taylor bubble.

$$
m=1.34 R C a^{\frac{2}{3}}
$$

Here $C a$ represents the capillary number $(C a=\mu U / \sigma)$, where $\mu$ is the dynamic viscosity, $U$ is the bubble velocity, $\sigma$ is the surface tension, and $R$ is the radius of the tube.

The theory of Bretherton [9] was recently modified and extended by Klaseboer et al. [11]. They found that the thickness of the liquid layer $(m)$ may reach $(\approx 1 / 3)$ of the tube radius at $C a>2$.

Gawusu and Zhang [12] performed a numerical study of the Dynamics of Taylor bubble by taking into consideration the presence of the tiny dispersed bubbles in the wake of Taylor bubble. Tomiyama et al. [13] performed a numerical simulation of bubbly flow including Taylor bubble. They predicted the formation of bubble wakes and complicated nature of bubble coalescence.

Baumbach et al. [14] appreciated the balance between the drag and buoyancy forces in their equation (3.1) of motion of the Taylor bubble in pipes. Under certain flow conditions, the liquid slug length $L_{f}$ region contains small spherical bubbles (Brauner and Ullmann [15], Pinto et al. [16]).

The aim of the present work is to present an analytical solution for the drag forces during the rise of Taylor bubbles in stagnant liquids in pipes. Most of the reported studies on Taylor bubble were based on numerical simulations, therefore the present investigation fills a gap in the literature due to its analytical nature. 


\section{Theoretical Analysis}

\subsection{Flat-ended Bubble with $\mathrm{Ca}<0.005$}

Klaseboer et al. [11] results showed that Bretherton's Eq. (3) is applicable at $\mathrm{Ca}<0.005$. The volume of liquid displaced by Taylor bubble is given as follows [12] (Figure 1b and Figure 2)

$$
V_{1}=\pi(R-m)^{2}\left[L_{g}-(R-m)\right]+(2 / 3) \pi(R-m)^{3}
$$

The second term here represents the volume of the hemisphere forming the bubble nose.

For $C a<0.005$, Eq. (3) gives very small values of the liquid layer $(\mathrm{m})$, hence we may say that $(R-m) \approx R$, and Eq. (4) becomes the following

$$
V_{1}=\pi R^{2}\left[L_{g}-R\right]+(2 / 3) \pi R^{3}
$$

e buoyancy force is given by the following

$$
B=\rho g V_{1}
$$

The classical definition of the drag force on the surface of the bubble or any other object in the flow field, allows us to write the drag as follows

$$
D=0.5 \rho U^{2} \pi(R-m)^{2} C_{D}
$$

For small values of $m$ as stated earlier, this equation becomes the following

$$
D=0.5 \rho U^{2} \pi R^{2} C_{D}
$$

Under steady rise of the Taylor bubble, the buoyancy force becomes equal to the buoyancy force, particularly when the bubble weight is neglected as it is too small to affect the equality between drag and buoyancy. Accordingly Eq. (6) is equal to Eq. (8) and we get the following

$$
\rho g V_{1}=0.5 \rho U^{2} \pi R^{2} C_{D}
$$

From this equation, the drag coefficient becomes

$$
C_{D}=\frac{\rho g V_{1}}{0.5 \rho U^{2} \pi R^{2}}
$$

Substituting Eqs. (1) and (5) into this equation and simplifying, we get the following

$$
C_{D}=8.16 \frac{L_{g}}{R}-2.72
$$

This equation represents the drag coefficient of a flat-ended Taylor bubble rising in a tube of radius $R$. 


\subsection{Round-ended Bubble with $\mathrm{Ca}<0.005$}

The volume of the bubble in this case (Figure 1a) becomes the following

$$
V_{2}=\pi(R-m)^{2}\left[L_{g}-2(R-m)\right]+(4 / 3) \pi(R-m)^{3}
$$

By applying the same procedure as that of Section 2.1, we get the following

$$
C_{D}=8.16 \frac{L_{g}}{R}-5.44
$$

\subsection{Flat-ended Bubble with Ca>2}

According to Klaseboer et al. [11] the liquid layer around the cylindrical portion of Taylor bubble may reach $(\approx 1 / 3)$ of the tube radius at $C a>2$ (see their Figure $3 b$ ). Therefore, $(R-m) \approx R-$ $(1 / 3) R=(2 / 3) R$. In this case, the bubble volume becomes the following

$$
V_{3}=\pi((2 / 3) R)^{2}\left[L_{g}-(2 / 3) R\right]+(2 / 3) \pi((2 / 3) R)^{3}
$$

By applying the same procedure as that of Section 2.1, we get the following

$$
C_{D}=8.16 \frac{L_{g}}{R}-1.812
$$

\subsection{Round-ended Bubble with Ca>2}

Equation (12) is used in the equality of drag to buoyancy as done before, but with $(R-m) \approx$ $R-(1 / 3) R=(2 / 3) R$ to get the following

$$
C_{D}=8.16 \frac{L_{g}}{R}-3.628
$$

\subsection{Flat-ended Bubble with $0.005<C a<2$}

With reference to Figure $3 \mathrm{~b}$ of Klaseboer et al. [11], we may choose any value of Ca within the above range, say $\mathrm{Ca}=0.5$, hence $m / R=0.22$ and $(R-m)=0.78 R$. This value may be substituted into Eq. (14) to get the following

$$
V_{4}=\pi(0.78 R)^{2}\left[L_{g}-(0.78) R\right]+(2 / 3) \pi(0.78 R)^{3}
$$

By applying the same procedure as that of Section 2.1, we get the following

$$
C_{D}=8.16 \frac{L_{g}}{R}-0.918
$$

By adopting the procedure mentioned above, we should be able to get a range of drag coefficients covering the range of Ca mentioned above for both types of Taylor bubbles of Figure 1. Figure 3 shows the variation of the drag coefficient versus the ratio $L_{g} / R$ for Eqs. (11), (13), (15), and (16). 


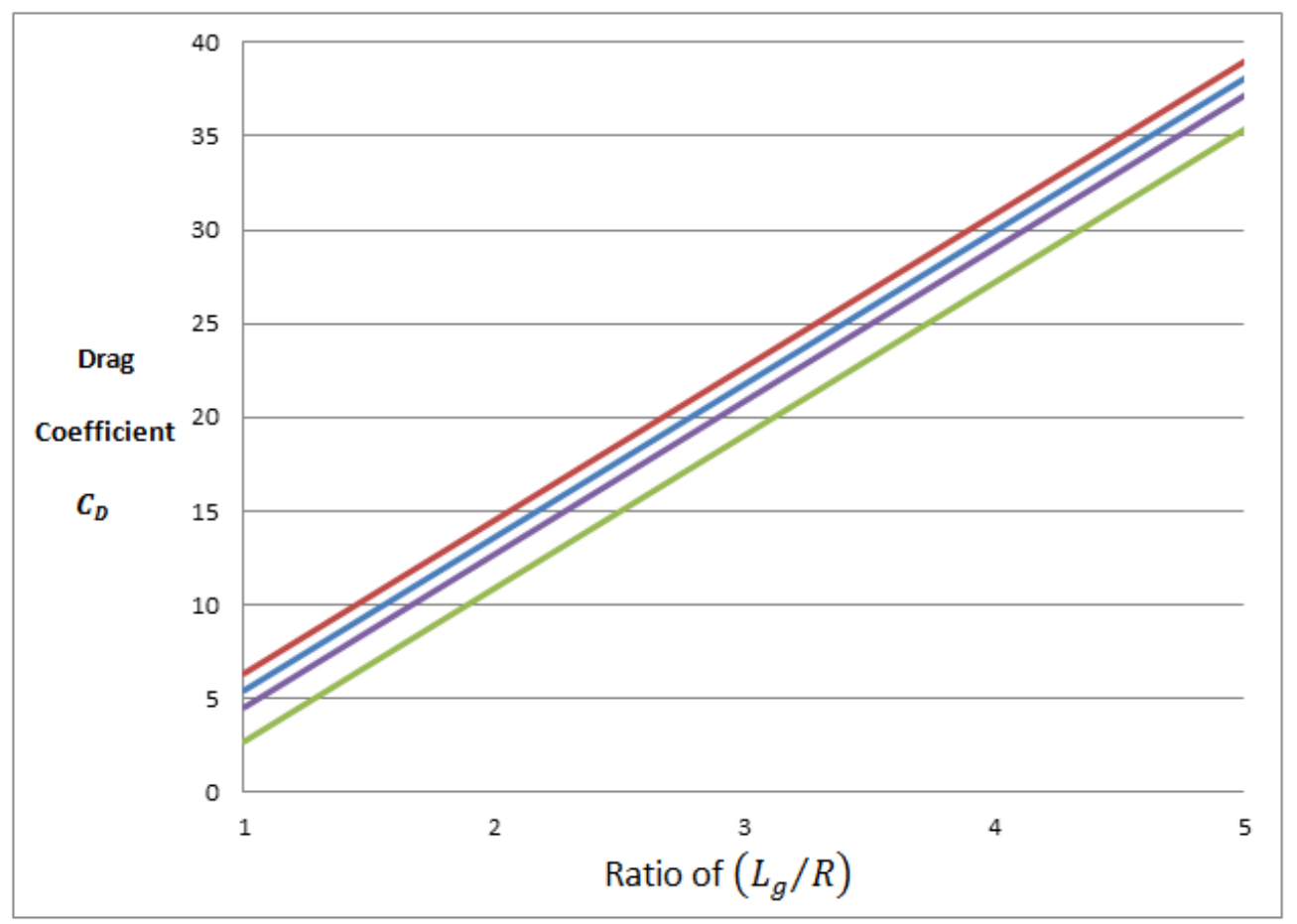

Figure 3 The variation of the drag coefficient versus the ratio $L_{g} / R$ for the following: red Eq. (15); blue Eq. (11); violet Eq. (16); and green Eq. (13).

Figure 3 shows that the stream-lined round-ended bubbles of Figure 1a (Eqs. (13) and (16)) encounter less resistance during its upward rise in the pipe, hence the drag coefficients are smaller than those of the flat-ended bubbles of Figure $1 b$ (Eqs. (11) and (15)).

\section{$2.6 C_{D}$ as a Function of Gas Volume Fraction}

In two-phase flow, engineers prefer the dependence of the $C_{D}$ on volume fraction $\alpha$ that can be obtained as follows (Figure 2)

$$
\alpha=\frac{\text { volume of Taylor bubble }}{\text { Total volume of bubble and liquid slug }}
$$

This ratio is done on a finite length along the pipeline, in this case $\left[L_{g}+L_{f}\right]$.

\subsection{1 $C_{D}$ at $C a>2$ for Flat-ended Bubble}

Following Figure 2 and substituting Eq. (14) into (19) yields

$$
\alpha=\frac{\pi((2 / 3) R)^{2}\left[L_{g}-(2 / 3) R\right]+(2 / 3) \pi[(2 / 3) R]^{3}}{\pi R^{2}\left[L_{g}+L_{f}\right]}
$$

Further simplification of this equation gives

$$
\alpha=\frac{(4 / 9)\left[L_{g}-(2 / 3) R\right]+(16 / 81) R}{\left[L_{g}+L_{f}\right]}
$$


This equation gives the ratio $\frac{L_{g}}{R}$ needed in our $C_{D}$ Eq. (15) as follows

$$
\frac{L_{g}}{R}=\frac{-\alpha\left(\frac{L_{f}}{R}\right)-(8 / 81)}{\alpha-(4 / 9)}
$$

To get positive values of $\frac{L_{g}}{R}, \alpha<(4 / 9)$

Akagawa et al. [17] developed the following correlation for the length of the liquid slug

$$
L_{f}=1.0 /\left(0.001 L_{g}^{0.55}+0.093 L_{g}{ }^{-0.52}\right)
$$

where $L_{f}$ and $L_{g}$ are in centimeters. Substituting this equation into Eq. (21) yields the following

$$
\alpha=\frac{(4 / 9)\left[L_{g}-(2 / 3) R\right]+(16 / 81) R}{\left[L_{g}+\left(1.0 /\left(0.001 L_{g}{ }^{0.55}+0.093 L_{g}{ }^{-0.52}\right)\right)\right]}
$$

The adoption of Akagawa et al.'s [17] correlation made us use the centimeter dimension for $L_{g}$, $L_{f}$ and $R$. Assuming $R=2.54 \mathrm{~cm}$, the above equation becomes the following

$$
\alpha=\frac{(4 / 9) L_{g}-0.249}{\left[L_{g}+\left(1.0 /\left(0.001 L_{g}^{0.55}+0.093 L_{g}{ }^{-0.52}\right)\right)\right]}
$$

A range of values of $L_{g}$ was used in this equation to get multiple values of $\alpha$ that were plotted and shown in Figure 4. This plot is useful for engineering applications, particularly where transparent pipes are used. A measure of the bubble length will be conducive to the local value of the volume fraction with the help of this plot for $R=2.54 \mathrm{~cm}$ and $\mathrm{Ca}>2$. We cannot go beyond the indicated range of $\alpha(0.25-0$.) because Taylor bubbles tend to coalesce and form annular flow in pipes (Delfos et al. [18]).

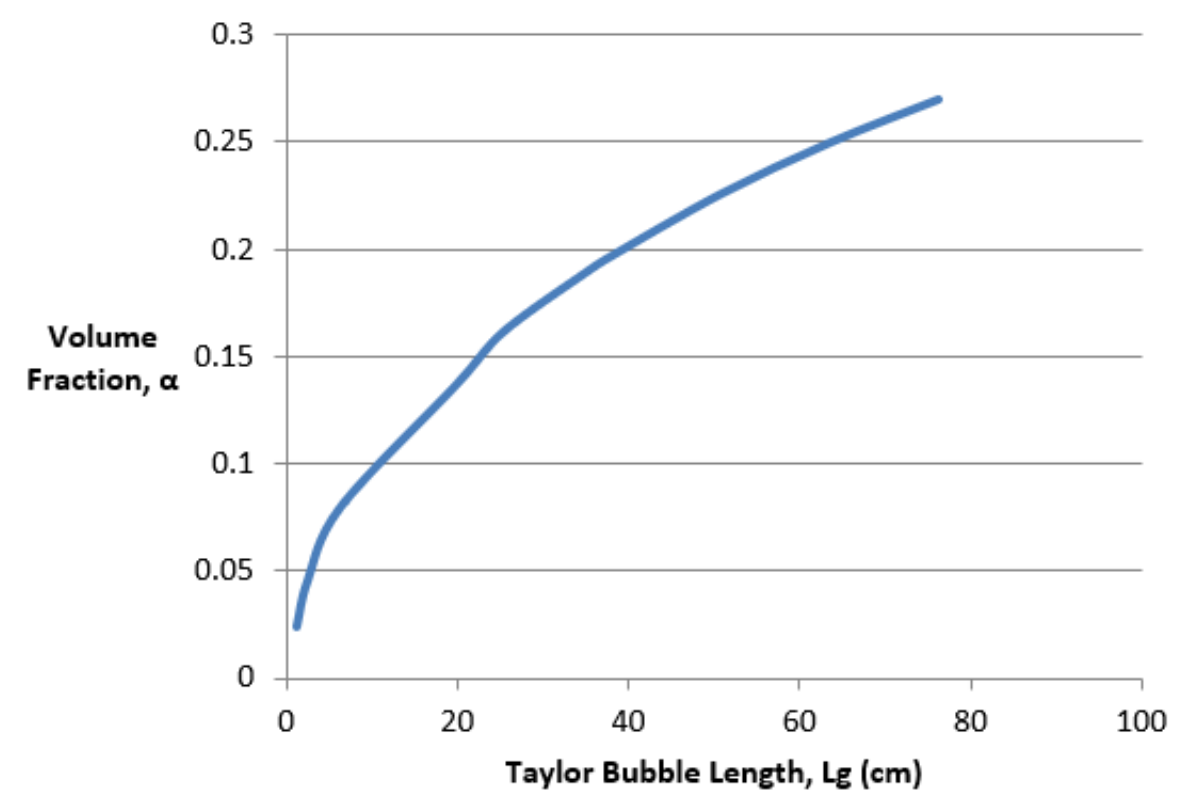

Figure 4 Gas volume fraction vs. bubble length for $\mathrm{R}=2.54 \mathrm{~cm}$ and $\mathrm{Ca}>2$. 
Substituting Eq. (22) into Eq. (15) to obtain $C_{D}$ as follows

$$
C_{D}=8.16\left(\frac{-\alpha\left(\frac{L_{f}}{R}\right)-(8 / 81)}{\alpha-(4 / 9)}\right)-1.812
$$

Values of $\alpha$ are obtained from Eq. (25) and plugged into this equation to get $C_{D}$ as shown by the blue curve in Figure 5.

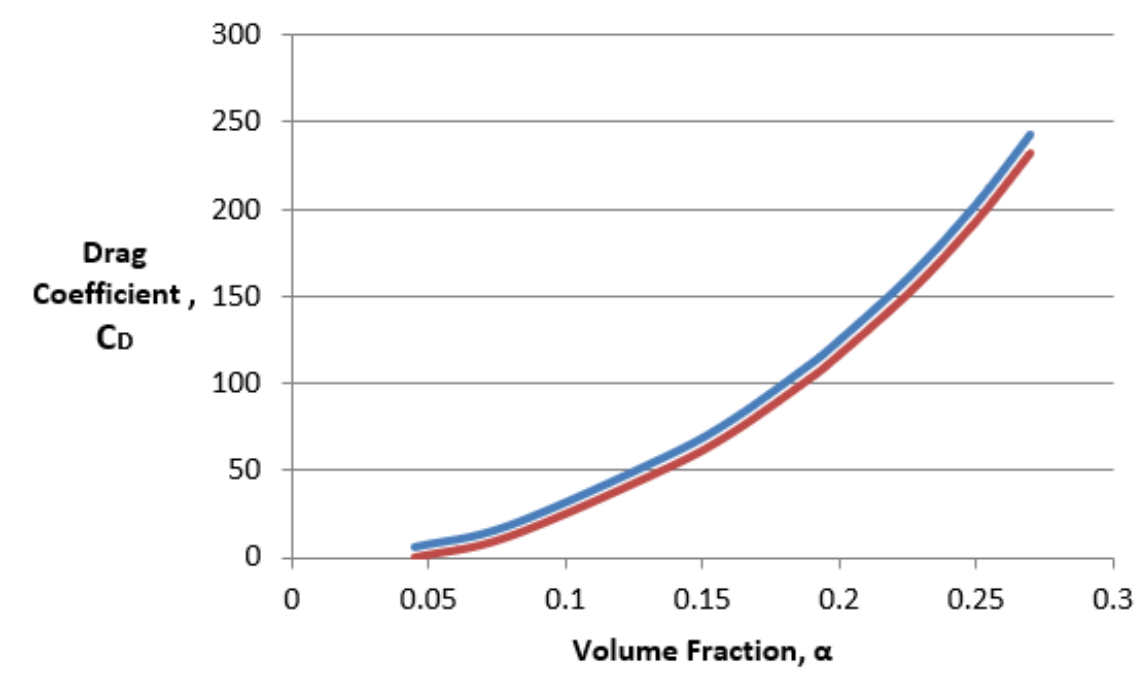

Figure 5 Drag coefficient vs. volume fraction for $\mathrm{R}=2.54 \mathrm{~cm}$ and $\mathrm{Ca}>2$; Blue for flatended bubble (Eq. 26) and red for round-ended bubble (Eq. (30)).

2.6.2 $\mathrm{C}_{\mathrm{D}}$ at $\mathrm{Ca}>2$ for Round-ended Bubble

In this case we substitute eq. (12) into eq. (19) to get the following

$$
\alpha=\frac{\pi(R-m)^{2}\left[L_{g}-2(R-m)\right]+\left(\frac{4}{3}\right) \pi[(R-m)]^{3}}{\pi R^{2}\left[L_{g}+L_{f}\right]}
$$

For $(R-m) \approx R-(1 / 3) R=(2 / 3) R$, this equation becomes the following

$$
\alpha=\frac{(4 / 9)\left[L_{g}-(2 / 3) R\right]+(32 / 81) R}{\left[L_{g}+L_{f}\right]}
$$

This equation gives the ratio $\frac{L_{g}}{R}$ needed in our $C_{D}$ Eq. (16) as follows

$$
\frac{L_{g}}{R}=\frac{(8 / 81)-\alpha\left(\frac{L_{f}}{R}\right)}{\alpha-(4 / 9)}
$$

Here also we employ Akagawa et al.'s [17] Eq. (23) and for $R=2.54 \mathrm{~cm}$, Eq. (28) becomes the following 


$$
\alpha=\frac{(4 / 9) L_{g}+0.2509}{\left[L_{g}+\left(1.0 /\left(0.001 L_{g}^{0.55}+0.093 L_{g}{ }^{-0.52}\right)\right)\right]}
$$

We apply Eq. (16) to get $C_{D}$ as function of $\alpha$ as follows

$$
C_{D}=8.16\left(\frac{(8 / 81)-\alpha\left(\frac{L_{f}}{R}\right)}{\alpha-(4 / 9)}\right)-3.628
$$

Values of $\alpha$ are obtained from Eq. (25) and plugged into this equation to get $C_{D}$ as shown by the red curve in Figure 5. Notice the smaller values of $C_{D}$ for the stream-lined round-ended bubble as compared to those of the flat-ended ones.

\section{Discussion}

The case of the rise of Taylor bubble in pipe is akin to the rise of the spherical-cap bubble in liquids under gravity. In both cases the bubble rise velocity is independent of liquid viscosity. For Reynolds number $>100$, the drag coefficient value approaches $C_{D}=2.7$, a value was obtained by many authors (e.g. Wegener and Parlange [19] and Joseph [20]).

Both the present results of the drag coefficient and that of Ishii and Mishima [5] are independent of liquid viscosity. There is a fundamental difference between the present results and that of Ishii and Mishima [5]. In the present results, the drag coefficient increases with the volume fraction contrary to that of Ishii and Mishima [5]. They attributed the decrease of the drag coefficient with respect to void fraction (See their Equation (2) above) as due to the entrainment of the small spherical bubbles in the wake of Taylor bubble. The present author did not consider the drag on these small bubbles, simply because their number is an ambiguity.

Kawaji et al. [21] and Tudose [22] obtained experimental drag data on a $23.4 \mathrm{~mm}$ diameter simulated flat-ended Taylor bubble made up from solid plastic and placed in a $25.6 \mathrm{~mm}$ inside diameter tube. Load cells were used to measure the drag forces on the surfaces of the solid simulated Taylor bubble. Two different lengths of $7.5 \mathrm{~cm}$ and $15 \mathrm{~cm}$ simulated Taylor bubbles were used. From the above data we calculated a liquid layer thickness of $0.11 \mathrm{~cm}$, hence we get $(R-m)=0.9141 R$. By following the method of calculation shown in section 2.5 we get the $C_{D}$ results shown by Table 1 . We should not expect close agreement due to the fact that the drag on a solid surface used by Tudose [22] is certainly greater than that on a bubble surface, but an agreement is existed when both Tudose [22] and us got greater $C_{D}$ for larger bubble length.

Table 1 Comparison with the experimental data of Tudose [22].

\begin{tabular}{llll}
\hline$L_{g}, \mathrm{~cm}$ & $\frac{L_{g}}{R}$ & Calculated $C_{D}$ & Experimental $C_{D}$ \\
\hline 7.5 & 5.86 & 36.33 & \\
15 & 11.72 & 77.8 & 66.7 (Figure 5.2.5) \\
\hline
\end{tabular}


Delfos et al. [18] produced a stationary Taylor bubble by introducing controlled amount of air into a down flow of water in pipe. They used two different grids to control the turbulence in the liquid flow. Grid B is at higher Reynolds number than grid A. Our results are compared with Delfos et al.'s [18] in Figure 6 where the agreement was not close but the trend is there, may be due to the indirect prediction of the volume fraction rather than direct measurement.

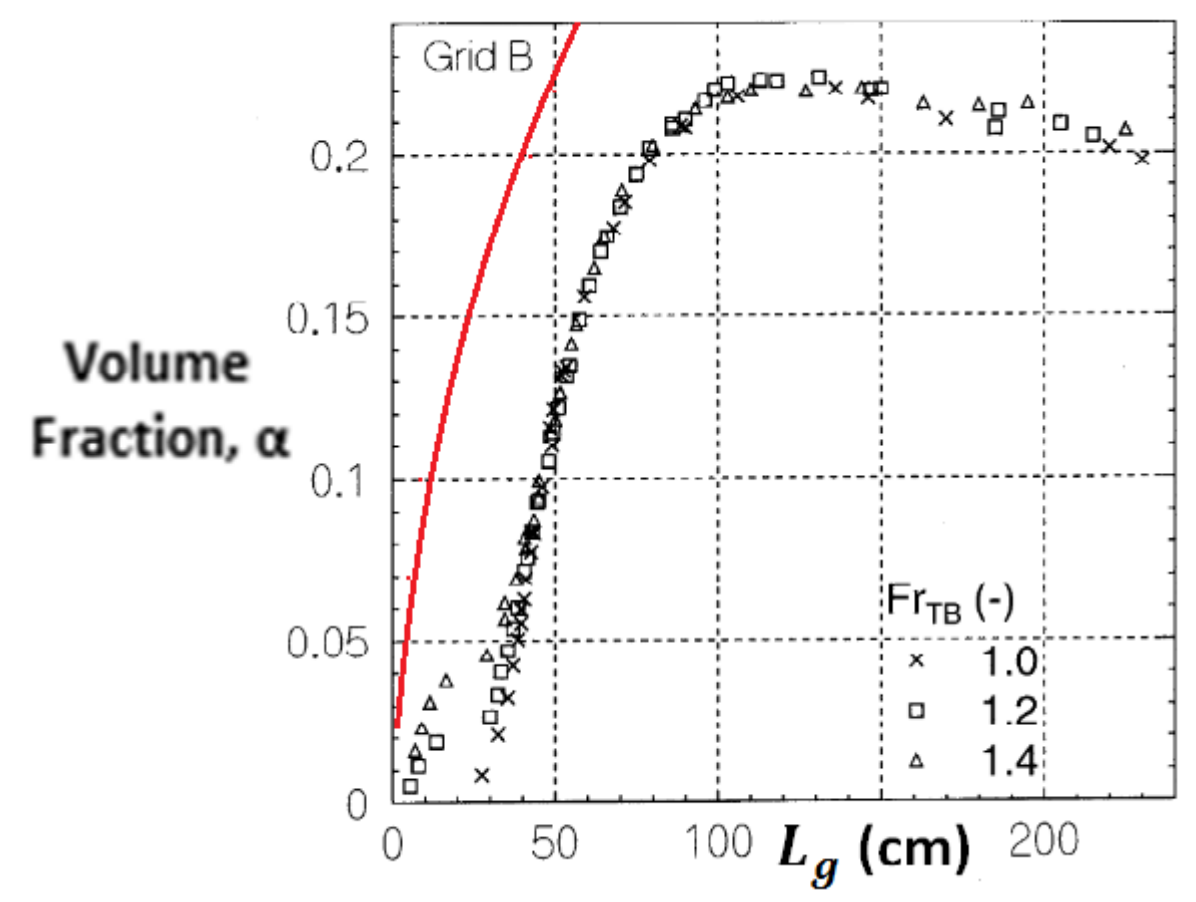

Figure 6 Comparison with the experimental data of Delfos et al. [18]. The continuous line is the present prediction.

\section{Conclusions}

Analytical solutions were obtained for the drag coefficients of a Taylor bubble rising in stagnant liquid in cylindrical pipes. The solution was based on a drag-buoyancy equality model. The derived drag coefficient equations were independent of liquid viscosity, but there are borders of applicability with viscosity dependence shown by the ranges of the capillary numbers used. The derived drag coefficient equations were functions of the geometry of the Taylor bubble and the gas volume fraction. The solutions were applicable at a wide range of Capillary numbers. The solution was validated by comparison with experimental data of other investigators.

\section{Author Contributions}

The author did all the research work of this study.

\section{Competing Interests}

The author has declared that no competing interests exist. 


\section{References}

1. US Nuclear Regulatory Commission. 0523-R504P-Westinghouse Advanced Technology [Internet]. Rockville: US Nuclear Regulatory Commission; 2011. Available from: http://pbadupws.nrc.gov/docs/ML1121/ML11216A098.pdf.

2. Campos JB, De Carvalho JG. An experimental study of the wake of gas slugs rising in liquids. J Fluid Mech. 1988; 196: 27-37.

3. Dumitrescu DT. Strömung an einer Luftblase im senkrechten Rohr. Z Angew Math Mech. 1943; 23: 139-149.

4. Davies RM, Taylor GI. The mechanics of large bubbles rising through extended liquids and through liquids in tubes. Proc R Soc Lond A Math Phys Sci. 1950; 200: 375-390.

5. Zukoski EE. Influence of viscosity, surface tension, and inclination angle on motion of long bubbles in closed tubes. J Fluid Mech. 1966; 25: 821-837.

6. White $\mathrm{ET}$, Beardmore $\mathrm{RH}$. The velocity of rise of single cylindrical air bubbles through liquids contained in vertical tubes. Chem Eng Sci. 1962; 17: 351-361.

7. Clanet $C$, Héraud $P$, Searby G. On the motion of bubbles in vertical tubes of arbitrary crosssections: Some complements to the Dumitrescu-Taylor problem. J Fluid Mech. 2004; 519: 359376.

8. Ishii M, Mishima K. Two-fluid model and hydrodynamic constitutive relations. Nucl Eng Des. 1984; 82: 107-126.

9. Kendoush AA. Hydrodynamic model for bubbles in a swarm. Chem Eng Sci. 2001; 56: 235-238.

10. Bretherton FP. The motion of long bubbles in tubes. J Fluid Mech. 1961; 10: 166-188.

11. Klaseboer E, Gupta R, Manica R. An extended Bretherton model for long Taylor bubbles at moderate capillary numbers. Phys Fluids. 2014; 26: 032107.

12. Gawusu S, Zhang X. Numerical simulations of the dynamics of Taylor bubble in the presence of small-dispersed bubbles. Heat Mass Transfer. 2021. Doi: 10.1007/s00231-021-03122-w.

13. Tomiyama A, Makino Y, Zun I. Numerical simulation of bubble flows using interface tracking and bubble tracking methods. In: Transactions on Modelling and Simulation. Billerica: WIT Press; 1999.

14. Baumbach V, Hopfinger EJ, Cartellier A. The transient behaviour of a large bubble in a vertical tube. J Fluid Mech. 2005; 524: 131-142.

15. Brauner N, Ullmann A. Modelling of gas entrainment from Taylor bubbles. Part A: Slug flow. Int J Multiph Flow. 2004; 30: 239-272.

16. Pinto AM, Pinheiro MC, Campos JB. On the interaction of Taylor bubbles rising in two-phase cocurrent slug flow in vertical columns: Turbulent wakes. Exp Fluids. 2001; 31: 643-652.

17. Akagawa K, Hamaguchi $H$, Sakaguchi T. Studies on the fluctuation of pressure drop in two-phase slug flow: 3rd report, pressure recovery behind a bubble, and lengths of bubble and liquid slug. Bull JSME. 1971; 14: 462-469.

18. Delfos R, Wisse CJ, Oliemans RV. Measurement of air-entrainment from a stationary Taylor bubble in a vertical tube. Int J Multiph Flow. 2001; 27: 1769-1787.

19. Wegener PP, Parlange JY. Spherical-cap bubbles. Annu Rev Fluid Mech. 1973; 5: 79-100.

20. Joseph DD. Rise velocity of a spherical cap bubble. J Fluid Mech. 2003; 488: 213-223.

21. Kawaji M, DeJesus JM, Tudose G. Investigation of flow structures in vertical slug flow. Nucl Eng Des. 1997; 175: 37-48. 
22. Tudose ET. Experimental investigation of Taylor bubble acceleration mechanism in slug flow. Toronto: University of Toronto; 1997.

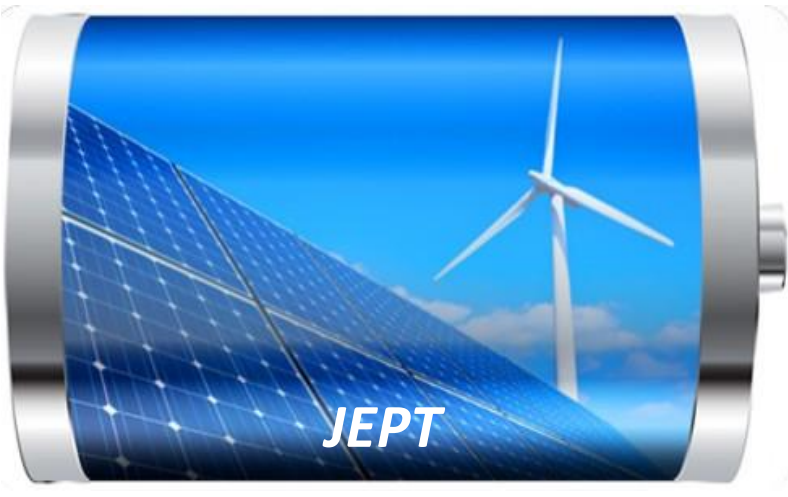

Enjoy JEPT by:

1. Submitting a manuscript

2. Joining in volunteer reviewer bank

3. Joining Editorial Board

4. Guest editing a special issue

For more details, please visit:

http://www.lidsen.com/journal/jept 